\title{
Diagnóstico e análise para a implementação do modelo PMS em uma indústria calçadista
}

Diagnosis and analysis for PMS model implementation in a footwear industry

Diagnóstico y análisis para la implementación del modelo PMS en una industria del calzado

\section{Marcia Athayde Moreira}

Doutora em Controladoria e Contabilidade pela Universidade de São Paulo - USP/SP Professora e Pesquisadora do Programa de Mestrado e Doutorado da Universidade da Amazônia - Unama

Endereço: Avenida Alcindo Cacela, 287, Bloco E, 4o. Andar. Umarizal

CEP: 66.060-902 - Belém/PA - Brasil

E-mail: marcia.athayde@unama.br

Telefone: (91) 4009-3018

\section{Everton Alain de Melo}

Mestre em Administração de Empresas na Faculdade Pedro Leopoldo

Doutorando em Engenharia de Produção na Universidade do Minho - Portugal

Endereço: Avenida Getúlio Vargas, 244. Centro

CEP: 36213-000 - Dores de Campos/MG - Brasil

E-mail: everton_melo1@hotmail.com

Telefone: (32) 3693-4027

Artigo recebido em 06/07/2015. Revisado por pares em 16/03/2017. Reformulado em 13/04/2017. Recomendado para publicação em 26/05/2017 por Carlos Eduardo Facin Lavarda (Editor-Chefe). Publicado em 31/08/2017. 


\title{
Resumo
}

Uma das opções que a literatura sugere para auxiliar desafios na avaliação de desempenho advém dos estudos dos sistemas de controle gerencial (SCG), que têm se mostrado um importante mecanismo para auxiliar na avaliação de desempenho dos processos de negócios. Diante desse fato, este estudo tem por objetivo analisar quais seriam as dificuldades e os possíveis benefícios que a implementação do modelo performance management systems - PMS de Ferreira e Otley (2009) poderiam oferecer para a avaliação de desempenho estratégico e operacional. Para tal, se realiza um estudo de caso em uma indústria de calçados de grande porte. Os resultados apontam que as principais dificuldades foram: identificação dos fatores chave de sucesso; identificação das medidas chave de desempenho; criação de uma política de recompensa ou sanção para os resultados. Já para os benefícios esperados, os resultados inferem que a implementação promoveria: maior integração entre a estratégia e as operações; a criação de estratégias de negócios mais consistentes; a medição correta do desempenho; e um melhor cumprimento dos objetivos traçados.

Palavras-chave: Sistema de Controle Gerencial; Performance Management Systems; Avaliação de Desempenho

\begin{abstract}
One of the options that the literature suggests assisting challenges in performance evaluation comes from studies of management control systems, which have been an important mechanism to assist in business performance evaluation. Given this fact, this study aims to analyze what are the difficulties and the possible benefits that the implementation of performance management systems - PMS model developed by Ferreira and Otley (2009) could help an evaluation of strategic and operational performance. So, we did a case study in a large Brazilian shoe industry. The results indicate that the main difficulties were: identification of key success factors; identification of key performance measures; creation of a reward or sanction policy for the results. As for the expected benefits, the results showed that the implementation would promote: greater integration between strategy and operations; creating more consistent business strategies; correct measurement of performance; and better fit with the objectives outlined.
\end{abstract}

Keywords: Strategy and operations; Management System Control; Performance Management Systems; Performance Evaluation

\section{Resumen}

Una de las opciones que la literatura sugiere para ayudar en la evaluación del desempeño provienen de estudios de los sistemas de control de gestión, que han sido una importante ayuda en la evaluación de los procesos de negocio. Ante este hecho, este estudio pretende analizar cuáles son las dificultades y los posibles beneficios que la implementación de lo modelo performance management systems - PMS preconizado por Ferreira y Otley (2009) podría proporcionar para la evaluación del desempeño estratégico y operacional. Para ello, fue realizado un estudio de caso en una gran industria brasileña del calzado. Los resultados indican que las principales dificultades fueron: identificación de los factores clave de éxito; identificación de las medidas clave de rendimiento; la creación de una política de recompensa o sanción causado por los resultados. En cuanto a los beneficios esperados, los resultados demuestran que la aplicación sería promover: una mayor integración entre la estrategia y las operaciones; la creación de estrategias de negocios más consistentes; correcta medición de desempeño; y mejor cumplimento con los objetivos trazados.

Palabras clave: Estrategia y operaciones; Sistema de Control de Gestión; Performance Management Systems; Evaluación del desempeño 


\section{Introdução}

Nos últimos anos, as discussões sobre o sistema de controle gerencial (SCG) tem ganhado maior relevância pelas dificuldades que os administradores têm enfrentado na condução dos processos de negócios (HERATH, 2007; MAGRETTA, 2012) e nesse sentido, a vantagem competitiva tem sido o foco das empresas que já alcançaram uma visão mais completa sobre as suas estratégias (PRAHALAD; HAMEL, 1990; MONTGOMERY; PORTER; 1998; TAVARES, 2000). Também tem se debatido muito sobre a importância de gerar estratégias de caráter disruptivo, uma vez que normalmente as estratégias geradas são de características incrementais (CANDIDO; 2011).

Nesse sentido, o sistema de controle gerencial - SCG se preocupa, precipuamente, em facilitar a implementação estratégica e influenciar o comportamento das pessoas (ANTHONY; GOVINDARAJAN, 2008). Para conseguir atingir esses objetivos, o SCG se vale do uso de instrumentos formais e informais de controle. Os instrumentos formais são indicadores, procedimentos, sistemas de informação entre outros. Já os mecanismos informais de controle possuem caráter mais intangível e podem ser representados pelo controle social onde indivíduos se auto ajustam ao padrão de interação de subgrupos da organização, e pelo controle cultural expresso pelos padrões normativos da organização que guiam o comportamento de seus funcionários, comumente identificados em declarações de missão, visão e valores (LEÃO; MELLO, 2010, p.254). Gomes e Salas (1999) corroboram com os últimos autores citados ao dizerem que:

O SCG se constitui no desenho de instrumentos e exercício de ações formais ou informais que busquem influenciar o comportamento das pessoas, manter a congruência levando em conta a cultura organizacional e o contexto social e competitivo (GOMES; SALAS, 1999, p.51).

Cabe ressaltar ainda a preocupação das empresas com seu SCG para assegurar transparência nos resultados e aumentar a credibilidade da empresa frente aos diversos stakeholders. Sousa Neto e Martins (2010) preconizam ainda sobre a necessidade de avaliação de desempenho de um negócio para acompanhar o quão eficientemente os ativos estão sendo utilizados para cumprir os objetivos dos acionistas.

Assim, dada a necessidade que os gestores possuem em avaliar o desempenho de suas estratégias e operações estabelecidas em seus processos de negócios, o objetivo deste estudo é analisar as dificuldades e vislumbrar os benefícios que incorreriam com a implementação do modelo performance management systems - PMS (sistema de gestão de desempenho) de Ferreira e Otley (2009).

A justificativa do estudo advém da necessidade, já exposta por Druker (1986) ao citar que a administração se preocuparia cada vez mais com a geração de resultados mensuráveis. Kindall e Gatza (1986) descrevem que o cerne da avaliação de um processo ou pessoa é a melhoria do desempenho, acreditando que o processo de avaliação de desempenho promove a melhoria dos resultados. Anthony e Govindarajan (2008, p.464) corroboram sobre a necessidade de buscar respostas para a avaliação de desempenho das empresas e para a implementação das estratégias ao dizerem que "[...] o mais importante aspecto do sistema de mensuração de desempenho é a sua capacidade de avaliar os resultados de maneira a levar a empresa a agir de acordo com a estratégia".

\section{Plataforma Teórica}

As primeiras discussões teóricas sobre os sistemas de controle gerenciais - SCGs tiveram início com Anthony nas décadas de 1960 e 1970. Suas abordagens enfatizavam o goal 
congruence (congruência de objetivos) por meio do processo de planejamento e controle. Anthony (1976, p. 21) definiu o SCG como "[...] o processo que utiliza a informação contábil para assegurar que os recursos são obtidos e aplicados de forma eficiente e efetiva na realização dos objetivos da empresa". Preconizou que o SCG é estruturado em planejamento, operações e análise de desempenho e precisa contemplar toda a empresa criando mecanismos que permitam gerenciar os processos visando assegurar que os recursos obtidos são utilizados de forma eficiente e eficaz para a realização dos objetivos da empresa.

Nesse contexto, os tópicos seguintes apresentam uma discussão acerca dos sistemas de controle gerencial no contexto da avaliação de desempenho, assim como apresentam os elementos dos sistemas de controle gerencial em suas dimensões desenho e uso.

\subsection{Sistema de Controle Gerencial e Avaliação de Desempenho}

Lorange e Morton (1974) antes de Anthony (1976), já iniciavam as discussões sobre SCG apontando que o SCG remete a ênfase de controles financeiros e orçamentos, reconhecendo a importância desses pontos, naquela época já identificando que medidas financeiras não serão suficientes para uma gestão adequada da empresa. Lorange e Morton (1974) ressaltam ainda a importância de identificar as variáveis de controle e o estabelecimento de vínculos entre essas e os planos de curto prazo (operacionais) e desses com os planejamentos de longo prazo (estratégicos).

O SCG precisa estar delineado pelas metas e objetivos, estrutura organizacional, a tecnologia disponível e o ambiente externo. Variáveis-chave são medidas que refletem os objetivos da empresa como exemplo: market share, inovações e EBITDA. Lorange e Morton (1974) chamam atenção para três tendências evolutivas do SCG. A primeira remete à necessidade de uma articulação mais próxima entre o SCG e o planejamento formal. A segunda remete a falta de estabilidade do ambiente externo que exigirá um conjunto mais robusto de variáveis de controle. A terceira ressalta que o aumento da diversificação das grandes empresas resultará em estruturas organizacionais mais complexas.

Contradizendo as abordagens de Lorange e Morton (1974) e Anthony (1976), Hofstede (1978) fez duras críticas ao modelo cibernético e racionalista dizendo que o modelo de SCG amparado em orçamento, planejamento e gestão por objetivos estava fadado ao fracasso. Hofstede (1978) criticou estruturas e processos de controle que atuam no ciclo de estabelecimento de metas, medição, comparação e trabalho sob os desvios encontrados (atuação diagnóstica) entendendo que esse modelo provoca a resistência dos colaboradores, pois as pessoas têm dificuldades em serem controladas a todo tempo. No entanto, Hofstede (1978) concordou com Lorange e Morton (1974) em dizer que o SCG não poderia mais estar alicerçado somente em medidas financeiras, sugerindo que um SCG deveria levar em conta também variáveis políticas, culturais e os relacionamentos das pessoas e suas negociações.

O final da década de 1980 e início da década de 1990 foram marcados por uma efervescência nas discussões sobre a relevância da contabilidade gerencial, assim como a do SCG. Kaplan (1991) e Otley (1999) discutem a necessidade de aperfeiçoamento da contabilidade de gestão frente à mudança do ambiente de negócios. Nessa época foi publicado o polêmico artigo Relevance lost: the rise and fall of management accounting pelos professores Johnson e Kaplan (1987). Gomes e Salas (1999) corroboram com tal preocupação ao postularem a necessidade urgente de um novo modelo de controle de gestão. Os autores confirmam as provocações feitas por Johnson e Kaplan (1987) sobre a perda de relevância da contabilidade gerencial. Autores contemporâneos como Gomes e Salas (1999) batizaram a abordagem como perspectiva psicossocial do controle, por contar com conhecimentos de outras áreas como psicologia, antropologia e a filosofia na construção do SCG propondo um modelo 
que tenha de caráter homeostático, onde o controle existe por si de forma autorregulada, sustentando o modelo em coalização com propósitos empresariais, negociação como regra e correção dos desvios durante a operação (GOMES; SALAS, 1999).

Otley (1999) faz também uma reflexão e provocação quanto ao desenvolvimento das teorias de SCG. Primeiro ele indaga se os contadores têm acompanhado a nova necessidade gerencial das empresas ou se ainda estão presos em técnicas de cálculos de custos. Ele afirma ser essencial que contadores que pretendem criar um SCG precisam desenvolver conhecimento do negócio. Em segundo, Otley (1999) afirma ainda que os sistemas de controle precisam refletir os objetivos de uma empresa. Por fim, é preciso olhar o contexto externo da empresa e o nível de valor que está sendo oferecido aos seus clientes.

Esse período de discussões colaborou para a evolução da teoria dos SCGs. Ao longo de 40 anos após as primeiras obras, muitos trabalhos, pesquisas e estudos de casos foram realizados sobre o tema proporcionando essa evolução. Os trabalhos que surgiram a partir da década de 1990 já estavam mais voltados para facilitar a implementação estratégica, as medidas não financeiras já estavam inseridas nos SCG e muito já se discutia sobre a importância de estabelecer vínculos entre operações e estratégias (FERREIRA; OTLEY, 2009). O SCG passava a exercer papel de maior importância nas empresas. $\mathrm{O}$ crescimento da concorrência, $\mathrm{o}$ advento da internet e a busca de profissionalização por parte dos gerentes também contribuíram para o processo de avanço e consolidação da teoria de SCG.

Alinhado com esta nova visão sobre o SCG, Flamholtz (1996) concordou com Anthony (1976) ao defender que o SCG deve, sobretudo, influenciar o comportamento das pessoas para que a empresa possa cumprir seus planos e alcançar seus objetivos, numa perspectiva que entende o processo de controle em quatro questões centrais: natureza do controle, funções gerenciais do controle, estrutura organizacional e customização do SCG, sendo a última de maior ênfase. Entretanto é possível perceber que a perspectiva do SCG de Flamholtz (1996) também trouxe em sua essência sinais das proposições de Hofstede (1978) com preocupações sobre o comportamento humano. Primeiramente, como a motivação e influência para que as pessoas ajam em congruência com os objetivos organizacionais. Em segundo, na integração dos esforços de forma eficiente e eficaz para o cumprimento destes objetivos, em concordância com Anthony (1976). O terceiro ponto é a avaliação de desempenho que levará os indivíduos a realizarem suas atividades dentro das metas estabelecidas, pois estes saberão que se agirem de forma diferente, isto não ficará oculto. Por fim, o quarto ponto recaiu sobre a necessidade do SCG de ser um mecanismo facilitador da implementação estratégica (FLAMHOLTZ, 1996).

Flamholtz (1996) ressalta ainda que uma empresa pode ter procedimentos de controle e não ter um SCG. Defende que um SCG é formado por três elementos principais: estrutura cibernética composta por quatro subsistemas (planejamento, operações, medição e avaliação e recompensa), estrutura da empresa e a cultura organizacional.

Outra importante contribuição que Flamholtz (1996) apresentou é uma descrição sobre a importância de se estabelecer vínculos entre planejamento estratégico, operações e avaliação de desempenho. Merchant e Van der Stede (2001) complementam sobre a importância do controle operacional e do controle estratégico e as causas comuns do primeiro não apoiar o segundo. Estas causas foram identificadas como falta de direção, problemas de motivação e limitações pessoais dos envolvidos.

Essa última perspectiva apresentada oferece pelo menos três contribuições para os gestores da empresa. Primeiro, o modelo esclarece a importância de monitorar as operações verificando se as mesmas estão em congruência com os objetivos. Em segundo, o modelo de SCG proposto por Flamholtz (1996) permite avaliar a eficácia dos sistemas estratégicos e operacionais. Por fim e em terceiro, os resultados apontam necessidades de melhorias e redesenho do SCG. 
Por sua vez Anthony e Govindarajan (2008) entendem e apresentam uma perspectiva ampliada do SCG como um sistema de integração do planejamento estratégico, preparação de orçamentos, alocação de recursos, mensuração de desempenho, avaliação e recompensa, alocação de centros de responsabilidade e preços de transferência. O SCG está fortemente vinculado à natureza de implementação estratégica, pautado aos fatores críticos de sucesso. Para esses autores o controle interativo é essencial para a sobrevivência da empresa criando uma empresa de aprendizado. A definição de empresa de aprendizado é expressa por Anthony e Govindarajan (2008, p.471):

Empresa de aprendizado efetivo é aquela em que funcionários de todos os níveis avaliam o ambiente, identificando potenciais problemas e oportunidades, trocando informações abertamente, e experimentando modelos alternativos de negócios para adaptar o ambiente emergente com sucesso (ANTHONY; GOVINDARAJAN, 2008, p.471).

Anthony e Govindarajan (2008) defendem ainda o que o principal objetivo do controle interativo é facilitar o desenvolvimento da empresa de aprendizado. Muitas decisões da empresa acontecerão informalmente de forma interativa por memorandos, reuniões, conversas e até expressões faciais. Anthony e Govindarajan (2008) fazem um complemento ao dizer que o modo do sistema de controle também é influenciado pelo diretor-presidente podendo ser pessoal ou impessoal, rígido ou flexível de acordo com o estilo da liderança. A abordagem de Anthony e Govindarajan (2008) ilustra o processo evolutivo da teoria de controle gerencial, partido de uma visão racionalista com um grande foco no controle das operações e dos indivíduos passando para um estado de considerável complexidade onde o controller precisa ter, além do conhecimento das medidas financeiras, entendimento sobre análise de ambiente, cadeia de valor e estratégias.

\subsection{Elementos do SCG: as Dimensões Desenho e Uso do SCG}

Flamholtz (1996) iniciou as discussões sobre os elementos que estruturam um SCG: desenho e uso. O primeiro (desenho) é tratado como regras, técnicas e procedimentos expressos em orçamentos, avaliação de desempenho entre outras ferramentas. Já o controle gerencial é apresentado com uma relevância muito maior. $\mathrm{O}$ foco não está propriamente no controle, mas no modo que o sistema é usado na influência do comportamento dos indivíduos.

Em outra perspectiva, Gomes e Salas (1999) tratam sobre os tipos de desenho do SCG mais adequado para cada empresa levando em conta sua dimensão (porte, tamanho) e o contexto social. Estas características são transmitidas ao sistema devido a fatores contingenciais que influenciam o sistema tais como estrutura da empresa, ambiente externo, governança, contexto social e cultural entre outros. Ressaltasse que esses fatores no futuro, seriam propostos como os elementos da dimensão desenho por Malmi e Brown (2008).

Segundo Ferreira e Otley $(2005 ; 2009)$, a estrutura do SCG é formada por dois constructos: desenho e uso. A dimensão desenho está relacionada com as características da informação e as técnicas de gestão que serão utilizadas enquanto a dimensão uso expressa como será o modo que os gestores irão usar das informações para a tomada de decisões. Também é possível dizer que a dimensão desenho é o elemento do sistema de controle gerencial que contempla as técnicas ou artefatos de contabilidade gerencial que serão utilizados no processo de controle. Mendes et al. (2002) conceituam que:

O desenho compreende a estrutura do controle e o processo de controle. A estrutura de controle deve considerar os indicadores (variáveis-chave) que possibilitarão o 
controle de cada unidade, a mensuração de sua atuação e o sistema de informação que permita o acompanhamento periódico da evolução dos indicadores identificados. Já o processo de controle representa o planejamento, a avaliação do resultado e as decisões corretivas (MENDES et al., 2002, p. 4).

Em uma abordagem diferente, Frezatti et al., (2009) têm definido que o desenho do SCG é formado pelas características da informação e técnicas que serão utilizadas. Tais características da informação seriam escopo, tempestividade, agregação, integração e custo. Quanto as técnicas, Ferreira e Otley (2005), Anthony e Govindarajan (2008), Frezatti et al. (2009) concordam que as mesmas são divididas em tradicionais, que podem ser exemplificadas por controle orçamentário, custeio por absorção e medidas financeiras, e por sua vez as técnicas contemporâneas são representadas pelo balanced scorecard, benchmarking e o economic value aded (EVA).

Ainda é possível citar a proposição de desenho do SCG proposta por Malmi e Brown (2008). Esses autores entendem que é possível concentrar os instrumentos de controle em quatro tipos de pacotes. Esses pacotes seriam constituídos por controles culturais, cibernéticos, de planejamento e controles administrativos.

Passando a discussão para a dimensão uso, Frezatti et al., (2009) postulam que a dimensão uso do SCG remete ao modo que os executivos utilizarão das informações geradas pelo sistema podendo ser diagnóstico, interativo e disfuncional. Simons (1987, 1990 e 1994) evidencia como o uso SCG deve estar preocupado com a criação de vantagens competitivas e implementação de estratégicas, discutindo a necessidade de os controles passarem de diagnósticos (fatos acontecidos) para interativos (presente para desenhar o futuro). O modelo de alavancas de controle desenvolvido por Simons (1994) é construído sobre quatro plataformas que ele denomina como alavancas do processo de controle resultando uma evolução do desenho e uso do controle gerencial. As premissas são: i) uso do SCG como sistema de crenças origina o direcionamento estratégico respeitando os valores; ii) uso do SCG como sistema de restrições delimita o campo de ação criando limitações, iii) uso do SCG como sistema de controle diagnóstico avalia a execução da estratégia por comparação com as metas; iv) uso do SCG como sistema de controle interativo trata das incertezas estratégias estimulando o debate e diálogo.

\subsection{O Modelo Performance Management Systems - PMSs}

Ferreira e Otley (2009) propõem um modelo de desenho e uso do SCG orientado para a avaliação de desempenho. Tal modelo foi batizado por seus criadores de modelo performance management systems - PMSs (sistema de gestão de desempenho) que busca proporcionar uma visão mais holística do negócio levando em conta o contex to e a cultura. O modelo é estruturado em: visão e missão, fatores-chave de sucesso, estrutura organizacional, planos estratégicos, medidas de desempenho-chave, definição de metas, avaliação de desempenho, sistema de recompensas, fluxo de informações sistemas e redes, uso do $P M S$, e força e coerência dos resultados. De forma mais estruturada, o PMS busca integrar os seguintes pontos, conforme Figura 1.

Moro (2014) complementa ao dizer que o PMS de Ferreira e Otley (2009) tem a premissa de gerir o desempenho estratégico e operacional de uma empresa, o que concorda Gomes e Salas (1999). Moro (2014) descreve ainda o PMS como uma evolução dos mecanismos formais e informais de controle utilizados pela organização para transmitir objetivos chave e metas aos envolvidos, sistematizados para a obtenção da mediação do desempenho. 
Figura 1: Sistema de Gestão de Desempenho.

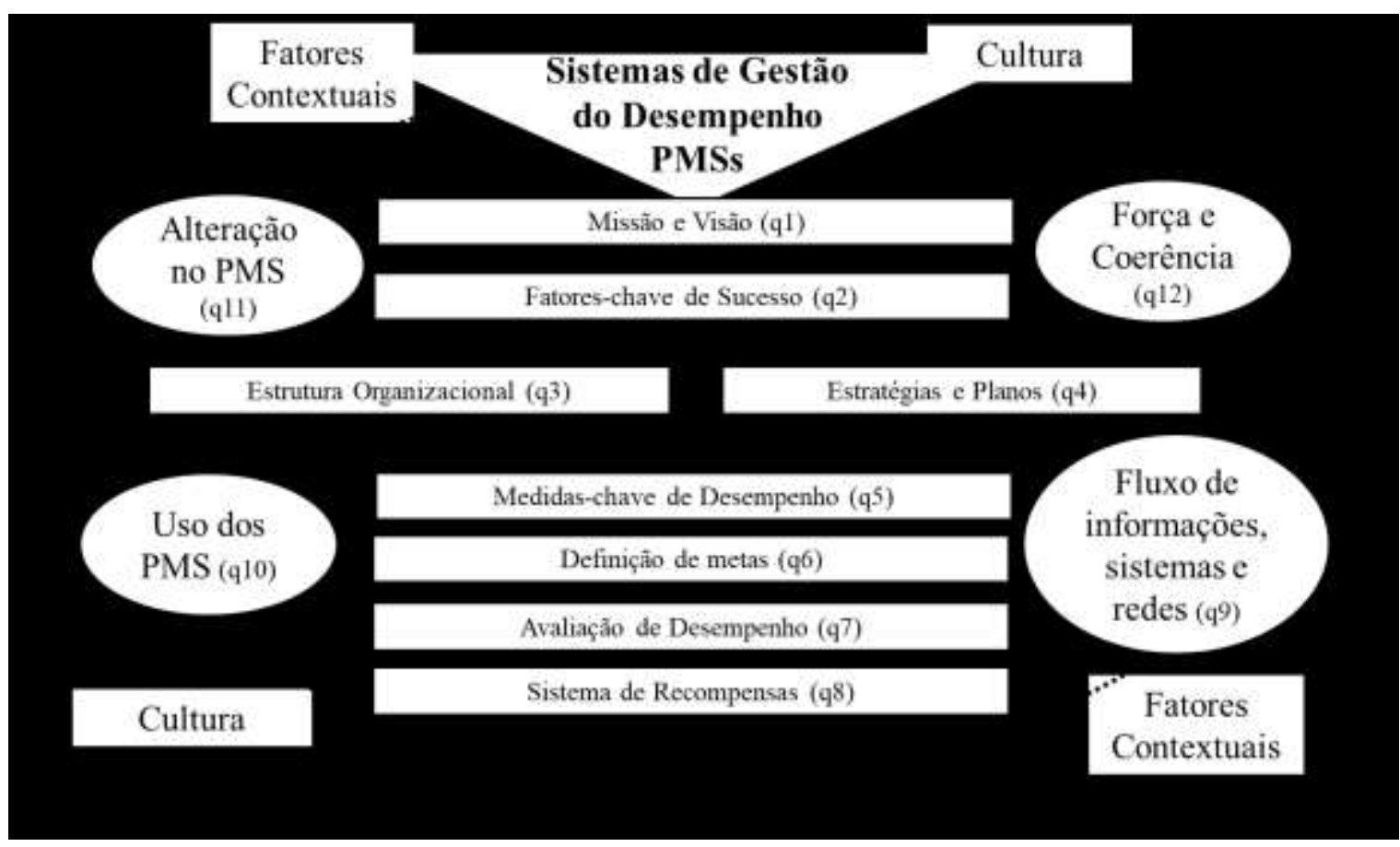

Fonte: MORO (2014).

O PMS é a base do extended framework for analysis (quadro ampliado de análise) de Ferreira e Otley (2009) que normalmente é o instrumento utilizado para a avaliação do desempenho empresarial quando se usa o modelo em discussão.

A estrutura do PMS se mostra promissora para apoiar o SCG a atingir seus principais objetivos: influenciar o comportamento dos indivíduos e facilitar a implementação das estratégias. O comportamento dos indivíduos é fortemente influenciado pelos mecanismos de controle (ANTHONY, 1976). Como o PMS é um modelo de avaliação de desempenho, os resultados do modelo permitem a aplicação da sugestão de Malmi e Brown (2008) quanto à necessidade da empresa criar uma política de recompensa. Para estes autores, a empresa precisa ter mecanismos de recompensa para resultados satisfatórios assim como mecanismo de punição para resultados insatisfatórios. Um processo de avaliação de desempenho promove um processo de accountability e este exerce influência sobre as pessoas da organização. Por sua vez, o processo de avaliação de desempenho também possibilita verificar durante o percurso o andamento dos planos estratégicos deliberados para um determinado período. Caso os resultados apontem que algumas das estratégias não estejam caminhando como esperado, é possível os gestores analisarem as causas e proporem ações que possibilitam uma melhor execução dos planos estratégicos definidos.

No contexto da empresa estudada, a necessidade de influenciar o comportamento dos indivíduos para que estes atuem em congruência com os objetivos organizacionais, somada a importância de se melhorar a implementação estratégica, tem sido temas de preocupação dos gestores e o $P M S$ oferece respostas que podem facilitar a solução destes desafios.

\section{Metodologia}

Para atingir os objetivos deste estudo, empreendeu-se uma pesquisa exploratória, de natureza qualitativa. Exploratória por que esse tipo de pesquisa proporciona maior 
familiaridade com o problema, permite a construção de hipóteses ou pressupostos e aproxima a visão geral de um determinado fato (COLLIS; HUSSEY, 2005). Quanto aos meios, a pesquisa é um estudo de caso em uma empresa industrial de calçados de segurança. Yin (2001) explica o que "um estudo de caso é uma investigação empírica de um fenômeno contemporâneo dentro de seu contexto de vida real".

Para a operacionalização da pesquisa, foi escolhida como unidade de análise uma indústria de calçados do estado de Minas Gerais. Esta escolha teve como motivos principais o acesso aos dados e o fato da empresa ser de grande porte, o que aumenta a chance de os resultados serem úteis para outras empresas. Além do que fora dito, era importante que a unidade de análise possibilitasse a observação dos sujeitos em suas rotinas cotidianas de trabalho para que não houvesse mudança de comportamento por parte desses no momento de coleta de dados, para confirmar o discurso com as práticas reais e assim tentar confirmar a aplicação empírica da teoria.

Por ser um estudo qualitativo, deve ser validado para maior credibilidade. Nesse sentido é importante a ligação entre os elementos da plataforma teórica e os achados da investigação empírica. Assim, foram utilizadas múltiplas formas de coleta de dados, permitindo a comparação dos resultados encontrados (MARTINS; THEÓPHILO, 2009). Para a realização da pesquisa empírica foram utilizados três métodos de coleta de dados: entrevistas, observação e análise de documentos, mediante estruturação de protocolo de pesquisa, conforme apresentado no quadro 1.

Quadro 1 - Protocolo para execução do estudo de caso

\begin{tabular}{|l|}
\hline $1^{\circ}$ Identificação da empresa participante do estudo \\
\hline Estudo das informações públicas sobre a empresa \\
\hline \\
\hline $2^{\text {o }}$ Reconhecimento da empresa \\
\hline Identificação do modus operandi da organização (observação direta das atividades) \\
\hline Obtenção e análise de documentos internos \\
\hline \\
\hline $3^{\circ}$ Realização das entrevistas (três níveis) \\
\hline Triangular entrevistas com os documentos e as observações \\
\hline Efetuar questionamentos adicionais aos entrevistados, se necessário. \\
\hline \\
\hline $4^{\circ}$ Analise dos dados obtidos \\
\hline Analisar o conteúdo dos materiais levantados com base nos objetivos da pesquisa \\
\hline Comparar achados empíricos com a teoria \\
\hline \\
\hline $5^{\circ}$ Apresentação de feedback aos participantes \\
\hline Entrega do trabalho final e apresentação dos resultados à empresa pesquisada \\
\hline Fonte: Elaborado com base em Alves (2010). \\
\hline
\end{tabular}

$\mathrm{Na}$ pesquisa documental foram analisados os documentos formais que descrevem os procedimentos de rotinas operacionais, documentos decorrentes do planejamento, com a descrição dos indicadores de controle da empresa e outros documentos que caracterizam o processo de gestão da empresa, como atas de reunião e relatórios de resultados.

O processo de observação permitiu a comparação dos resultados encontrados de forma empírica com a literatura na busca de conseguir verificar se as práticas sugeridas pelos autores do tema são evidenciadas nas rotinas da empresa, demonstrando as lacunas existentes entre a prática da empresa e a teoria. Este momento ajudou a melhor compreender o sistema de avaliação de desempenho do SCG da empresa para analisá-lo frente ao modelo PMS. 
Ademais, foram realizadas entrevistas com dois diretores da empresa (nível estratégico), com o controller (nível tático) e dois analistas, sendo um analista financeiro e outro analista de compras (nível operacional), visando obter informações relevantes para a análise dos dados, as entrevistas foram agendadas por telefone e realizadas presencialmente. Sobre o roteiro das entrevistas, foi baseado na teoria estudada, com ênfase nos elementos que envolveram a implantação do modelo PMS.

Por fim, foi realizado o processo de observação das rotinas da empresa. Nesse ponto cabe destacar que um dos autores é também colaborador da empresa, e dessa forma, durante o período de realização da pesquisa foi realizado um processo sistemático de observação das funções e realização de atividades pelos colaboradores internos, assim como a concordância das atitudes observadas com os planos e procedimentos previstos na empresa.

\section{Resultados}

A empresa em que o estudo de caso foi realizado é uma indústria de grande porte de calçados profissionais, com mais de quarenta anos de atividade e cerca de dois mil funcionários diretos. Nos últimos três anos a empresa tem passado por uma fase de expansão dos negócios marcado pela abertura de sete novas plantas de fabricação. Este crescimento trouxe preocupação para a direção da empresa quanto ao gerenciamento das atividades e com a transmissão da visão, missão e valores de forma alinhada com os objetivos da matriz. A empresa possui um SCG que evolui ao longo dos anos. Pode-se dizer ainda que as dimensões desenho e uso do SCG da empresa estão alinhadas com as proposições de Frezatti et al., (2009) e Ferreira e Otley (2005).

Para a operacionalização do estudo, foi elaborado um diagnóstico para evidenciar da forma mais clara possível, as prováveis dificuldades na implementação do modelo $P M S$. Em segundo momento, no mesmo diagnóstico, o objetivo foi evidenciar os benefícios que poderiam advir da implementação do modelo. Para tal, foram analisados os doze construtos do modelo PMS (os quais podem ser visualizados na Figura 1) frente ao contexto atual da empresa e seus fundamentos, tais como cultura, estrutura e SCG atual.

O primeiro ponto do $P M S$ é a identificação da missão e a visão da empresa. Os resultados encontrados na empresa corroboram a teoria. Devido à implantação da ISO 9000 que ocorreu nos anos 2000, a empresa precisou definir declarações de visão, missão e valores. Tais declarações são bem difundidas dentro da organização, conhecidos pelos diferentes níveis da empresa e atualmente e são geridas pelo escritório da qualidade, coadunando com a estratégia do modelo PMS de Ferreira e Otley (2009), que prevê uma visão mais holística do processo. Quanto a isso, a difusão da missão e visão em todos os níveis hierárquicos da empresa é o ponto-chave inicial que favorecerá a implementação dos demais elementos previstos no modelo PMS.O segundo ponto do $P M S$ está em identificar quais são os principais fatores compreendidos como o centro para o sucesso futuro da empresa (fatores de sucesso). Os resultados encontrados na análise empírica contradizem a teoria., pois a identificação dos fatores de sucesso não está clara na empresa. Nesse ponto resgata-se as ideias de Flamholtz (1996) que, dentre outras observações pertinentes, destacou a importância de se estabelecer vínculos entre planejamento estratégico, operações e avaliação de desempenho. Nesse sentido, a ausência da identificação dos fatores de sucesso é uma fragilidade encontrada no processo do desenho do SGC. Assim, seria necessário um estudo para identificar os fatores de sucesso e as vantagens competitivas.

Mapear a estrutura da empresa é o terceiro ponto do $P M S$. Os resultados corroboraram a teoria. A estrutura da empresa é bem definida atualmente. Existe divisão clara de funções e responsabilidades, planos de cargos e salários bem definidos e segregação de funções. Esse 
ponto favorece a implementação do $P M S$ e vão ao encontro das proposições de Hofstede (1978), que desde a década de 70 manifestou preocupações com o comportamento humano. A divisão de funções e responsabilidades e a segregação de funções favorece a motivação e a influência para que as pessoas ajam em congruência com os objetivos organizacionais, favorecendo a integração dos esforços para o cumprimento dos objetivos organizacionais (ANTHONY, 1976).

O quarto ponto trata da estruturação dos planos estratégicos definidos e os processoschave necessários para garanti-los. Os resultados encontrados confirmam em parte a teoria. Foi possível confirmar a existência de planos estratégicos na empresa. Estes foram definidos por um comitê formado por diretores, gerentes, equipe comercial e uma empresa de consultoria. Quando criados, o objetivo da empresa era que estes contemplassem os anos de 2014 a 2016. No entanto, uma análise crítica no plano criado permite concluir que as ações definidas estão mais relacionadas à melhoria contínua dos setores e suas atividades do que estratégias de negócios propriamente ditas. Isto pode estar ligado ao fato de que a empresa ainda está construindo conhecimento neste campo estratégico. Ainda analisando o processo de definição e construção das estratégias, as fragilidades aumentam quando se coloca em discussão os processos-chave necessários para garanti-las. Não existe definição de quais processos são essenciais para a implementação das estratégias. Pelo menos não para uma boa parte destas. Esse quarto ponto, assim como o segundo ponto analisado, prejudica a consolidação dos SCG a partir do modelo $P M S$, na medida em que é crítico no modelo PMS que a organização transmita objetivos chave e metas aos envolvidos, sistematizados para a obtenção da mediação do desempenho (MORO, 2014), o que deveria ser viabilizado a parir da definição dos planos estratégicos.

Dando sequência a estruturação do $P M S$, o quinto ponto se preocupa em estabelecer previamente a forma pela qual estes processos podem e serão medidos. Os resultados também confirmam parcialmente a teoria. Atualmente, a empresa possui uma reunião de controle e alinhamento com o planejamento estratégico mensal que não está bem alinhada com o planejamento estratégico formal definido para a empresa documentado. $\mathrm{Na}$ reunião do planejamento estratégico (que é uma reunião de acompanhamento dos controles mensais) são apresentados e discutidos os resultados de indicadores que não são estratégicos. O contrário também é verdadeiro, mediante a observação, foi constatado que durante a reunião algumas premissas estratégicas não são discutidas e não foram revisitadas.

Os sexto e sétimo pontos confirmam em parte a teoria e serão avaliados em conjunto. $\mathrm{O}$ sexto ponto de estruturação do $P M S$ é definir previamente os níveis de desempenho esperado para as operações e o sétimo ponto trata de definir se as avaliações serão objetivas, subjetivas ou mistas. Os resultados confirmam a teoria pelo fato de que todos os indicadores que a empresa tem definido possuem metas acordadas entre o gestor do indicador e a direção da empresa, e a empresa se vale somente de seus indicadores para avaliar seu desempenho. Pode-se dizer que o instrumento formal de controle e avaliação de desempenho é objetivo. Observa-se então que o desenho do SCG da empresa analisada está aparentemente bem delineado, mas é frágil no relacionamento com as estratégias (que não estão bem definidas). Quando observada a função uso, remete-se às observações de Simons (1987, 1990 e 1994) o qual evidencia que o uso SCG deve estar preocupado com a criação de vantagens competitivas e implementação de estratégicas, o que não se observa na empresa analisada.

A definição de políticas para recompensas ou sanções para o cumprimento ou não cumprimento das metas constitui o oitavo ponto de estruturação do PMS. Os resultados contradizem a teoria uma vez que este ponto precisa ser desenvolvido na empresa, que atualmente não está preparada para atender este elemento. Nesse sentido Malmi e Brown (2008) observaram a necessidade de a empresa criar uma política de recompensa para resultados satisfatórios assim como mecanismo de punição para resultados insatisfatórios, no entanto, por 
se tratar de uma empresa familiar e muito relacional, existe grande dificuldade em punir resultados insatisfatórios, sobretudo com os membros da família que compõem a diretoria.

O nono ponto de estruturação do $P M S$ remete em definir o canal de comunicação da estratégia e da avaliação de desempenho. A empresa está desenvolvendo um canal de comunicação que deve ser administrado pelo setor de endomarketing. Este canal poderia ser utilizado para a comunicação dos resultados. Quanto à avaliação de desempenho, o modelo de reunião atualmente utilizado poderia ser mantido com a realização de alguns ajustes. Tais resultados corroboram a teoria em parte. Mais uma vez se observa que a empresa está bem estruturada e seus processos e controles internos são claros e bem disseminados, há uma real intenção de promover um sistema de gerenciamento do desempenho e os elementos de estruturação são visíveis.

O tipo de uso da informação é a preocupação central do décimo ponto do PMS. O tipo de uso da informação na empresa está bem alinhado com as proposições de Frezatti et al., (2009). Os controles financeiro, de produção e de vendas, por exemplo, utilizam a informação de forma diagnóstica controlando e atuando sobre qualquer desvio dos resultados frente ao padrão esperado. Por sua vez os processos de planejamento estratégico, planejamento operacional, reuniões de diretoria e trabalhos de comitês possuem um uso da informação predominantemente interativa.

O décimo primeiro ponto do $P M S$ é estabelecer um procedimento para a alteração dos objetivos em função dinâmica com o ambiente. Como o mercado está cada vez mais dinâmico e instável, é de suma importância que os processos de negócio tenham flexibilidade e agilidade para se moldarem ao ambiente. Frezatti et al., (2009) destacam que o uso do SCG como sistema de controle interativo trata das incertezas estratégias estimulando o debate e diálogo. Os resultados levam a inferir que a empresa tem facilidade para trabalhar com procedimentos em seus processos e atividades. Não seria um dificultador na implantação.

Finalmente, quanto ao décimo segundo ponto de estruturação do $P M S$ este trata da análise da força e coerência do modelo adotado realizando ajustes, se necessário para fortalecer o modelo. As reuniões de análise de desempenho que são realizadas para discutir o processo do $P M S$ e os resultados do extended framework for analysis (quadro ampliado de análise) se mostram como o momento mais propício para analisar e discutir os benefícios do modelo, assim como a proposição de ajustes que se apresentarem necessários. O uso diagnóstico do modelo promove o uso interativo, e este por sua vez, possibilita a evolução e melhoria contínua da empresa por meio da análise e discussão do desempenho.

Visando uma melhor compreensão dos resultados encontrados, o quadro 2 sumariza para cada um dos doze que estruturam o PMS, e o diagnóstico realizado na empresa objeto de estudo, confrontando os achados com a proposta realizada por Ferreira e Otley (1999).

\subsection{Reflexão sobre as Dificuldades na Implementação}

Sobre os pontos evidenciados como uma ameaça para implementação do modelo $P M S$, o estudo de caso revelou outras situações de caráter interno que não foram inicialmente vislumbradas, mas se mostraram como dificultadores da implementação. O primeiro seria a baixa disponibilidade de pessoas capacitadas para a implementação. Isto não seria uma barreira, mas influenciaria no tempo para a conclusão do projeto uma vez que se levaria certo tempo para treinar e capacitar às pessoas quanto ao novo modelo. A cultura familiar da empresa se mostra um desafio quanto ao sistema de avaliação de desempenho. Existe grande dificuldade em recompensar benefícios assim como existem barreiras para penalizar resultados insatisfatórios, sobretudo quando possíveis resultados insatisfatórios são gerados por membros 
da família, ou ainda, quando os resultados ruins fossem gerados por pessoas de maior ligação pessoal aos membros da família.

Quadro 2: Situação atual da empresa para a implementação do modelo PMS

\begin{tabular}{|c|c|c|c|}
\hline $\mathbf{N}^{\mathbf{o}}$ & Elementos & Resultados & Observações \\
\hline 1 & Missão e Visão & Adequados & Pontos bem definidos atualmente na empresa \\
\hline 2 & Fatores Chave de Sucesso & Não adequados & Pontos não evidenciados. Necessitam ser trabalhados \\
\hline 3 & Estrutura Organizacional & Adequados & Ponto bem definido atualmente na empresa \\
\hline 4 & Estratégia e Planos & Não adequados & $\begin{array}{l}\text { Planos frágeis,mais alinhados a operações do que às } \\
\text { estratégias de negócios }\end{array}$ \\
\hline 5 & $\begin{array}{l}\text { Medidas Chave de } \\
\text { Desempenho }\end{array}$ & $\begin{array}{l}\text { Parcialmente } \\
\text { adequados }\end{array}$ & $\begin{array}{l}\text { Falta alinhamento entre as medidas de desempenho e } \\
\text { as estratégias }\end{array}$ \\
\hline 6 & Definição de Metas & $\begin{array}{l}\text { Parcialmente } \\
\text { Adequados }\end{array}$ & $\begin{array}{l}\text { A empresa trabalha com metas, ressalva-se a medida } \\
\text { da estratégia pelas operações. }\end{array}$ \\
\hline 7 & Avaliação de Desempenho & $\begin{array}{l}\text { Parcialmente } \\
\text { Adequados }\end{array}$ & $\begin{array}{c}\text { A empresa avalia seu desempenho, ressalva-se a } \\
\text { medida da estratégia. }\end{array}$ \\
\hline 8 & Sistema de Recompensas & Não adequados & Pontos não evidenciados. Necessitam ser trabalhados \\
\hline 9 & Fluxo de Informações & Adequados & $\begin{array}{c}\text { Existem canais que podem ser utilizados para } \\
\text { divulgas as informações do } P M S\end{array}$ \\
\hline 10 & Uso dos PMS & Adequados & $\begin{array}{l}\text { A empresa tem experiência em uso diagnóstico e } \\
\text { interativo das informações do SCG }\end{array}$ \\
\hline 11 & Alteração do PMS & - & $\begin{array}{l}\text { Não se mostra uma ameaça para o sucesso do } \\
\text { modelo na empresa }\end{array}$ \\
\hline 12 & Força e Coerência & - & $\begin{array}{l}\text { Não se mostra uma ameaça para o sucesso do } \\
\text { modelo na empresa }\end{array}$ \\
\hline
\end{tabular}

Fonte: elaborado pelos autores.

Outra preocupação seria com a mudança do sistema de medidas de desempenho, uma vez que o histórico da empresa remete a forte restrição a mudanças. Seriam encontradas barreiras com alguns diretores, gerentes e coordenadores que implantaram o sistema atual de desempenho, que defenderiam a permanência do modelo atual por questões mais pessoais do que técnicas. Além do que já fora dito, seriam necessárias adequações no sistema de informação da empresa para que este gerasse algumas informações essenciais ao $P M S$ que até o momento não existem na empresa. Para tal, o software precisaria de customizações, o que demandaria de investimentos financeiros além de tempo para o processo de desenvolvimento, testes e validação das novas rotinas.

\subsection{Reflexão sobre os Ganhos na Implementação}

É importante destacar também que a implementação do modelo oferece ganhos para o processo de gestão da empresa. Primeiramente se espera que o $P M S$ promova maior integração entre estratégia e operações. Os pontos 2, 4, 5, 6 e 7 estão fortemente relacionados ao controle de operações e na identificação da maneira que estes estão relacionados com as estratégias de negócios podendo potencializá-las e monitorá-las, o que está alinhado com as proposições de autores como Gomes e Salas (1999), Flamholtz (1996) e Magretta (2012). 
Esta integração entre estratégias e operações promove a elaboração de estratégias de negócios mais consistentes. Atualmente as estratégias da empresa têm sofrido uma alta taxa de insucesso (somente $41 \%$ das estratégias pretendidas nos dois últimos ciclos de planejamento foram implementadas) e isto se deve, em parte, a dificuldade de entender ao longo do período, se os esforços necessários para que a implementação aconteça estão sendo realizados a contento.

Outro ganho importante trata do avanço que a empresa passaria em seu processo de medição e avaliação do desempenho. A implementação do $P M S$ requisitaria dos gestores a entender claramente pontos de grade importância para o processo de negócios que hoje não estão claros na empresa como exemplo a vantagem competitiva, os fatores chave de sucesso e os sistemas de recompensa. O modelo atual de verificação do desempenho não espelha se a empresa tem sido eficiente em implementar seus planos estratégicos, sendo mais voltado para a análise do desempenho operacional.

Acredita-se que a soma destes pontos contribuirá para uma melhora no cumprimento dos objetivos traçados. As medições corretas evidenciarão melhor os pontos que precisam ser trabalhos. O sistema de recompensa e sanções ajudará a influenciar o comportamento das pessoas. O estabelecimento de vínculo e controle entre estratégias e operações ajudará a melhorar a congruência de objetivos e a sinergia entre os setores.

\section{Conclusões}

Devido à necessidade de as empresas avaliarem o desempenho de suas estratégias e operações para ajudar os gestores a conduzirem os processos de negócios, os estudos do SCG têm se esforçado em propor modelos e ferramentas que colaborem para contornar estes pontos. Um dos modelos que a literatura sugere é o $P M S$ de Ferreira e Otley (2009) que se estrutura em doze construtos para promover a avaliação de desempenho dos negócios.

Com isto, este estudo buscou primeiramente diagnosticar, considerando os parâmetros propostos por Ferreira e Otley (1999), o estado atual de desenvolvimento do sistema de gestão de desempenho da empresa escolhida para a análise, e concomitantemente entender quais serias as dificuldades e os benefícios que a implementação do modelo $P M S$ resultaria para essa empresa que pertence ao ramo industrial do segmento de calçados.

A metodologia utilizada foi o estudo de caso, e os dados foram coletados prioritariamente por meio de pesquisa documental, complementados por entrevistas não estruturadas e observação participante. Os resultados expandem o conhecimento sobre o tema e respondem a pergunta orientadora do estudo. Foi possível observar que dos doze pontos que estruturam o $P M S$, sete já estão adequados ou parcialmente adequados na empresa, facilitando o processo de implementação possibilitando confirmar, que na prática, a empresa vem adotando elementos de gestão que caracterizam um sistema de controle gerencial conforme preconiza a teoria. Estes pontos são: missão e visão, estrutura organizacional definida, processo de planejamento estratégico, existência de metas, processo de avaliação de desempenho, existência de fluxo de informações e uso diagnóstico e interativo por parte dos colaboradores. Entretanto, três itens: sistemas de recompensas, identificação de fatores-chave de sucesso e medidas sobre os fatoreschave não puderam ser implementados, contradizendo a teoria. Cabe ressaltar que outros dois itens: capacidade de alteração do $P M S$ e força e coerência do modelo não foram atendidos por serem processos que devem ser trabalhados após implementação, quando o sistema já estiver apresentando resultados aos gestores. Entretanto estes pontos não se mostraram como impeditivos da implementação.

As implicações gerencias do estudo se expressam na evidenciação de outras situações inicialmente não contempladas no projeto de implementação, mas que se mostraram de grande 
interferência no decorrer do processo: disponibilidade de recursos humanos, cultura familiar e resistência a mudanças. A observação destes pontos se mostrou tão importante quanto à atenção aos doze pontos estruturais do $P M S$.

$\mathrm{Na}$ medida em que os resultados apontam que as principais dificuldades encontradas seriam: identificar os fatores de sucesso; melhorar a qualidade dos planos estratégicos; suprimir barreiras de resistência a mudanças; falta de interesse para criação de um sistema de recompensas e resistência em alguns casos quanto ao sistema de sanções, se torna possível a elaboração de um plano de ações que colaborem com a mitigação dos problemas/riscos supracitados, e com isso, não colocar o projeto de implementação em risco. Já para os benefícios esperados, os resultados inferem que a implementação promoveria: maior integração entre estratégia e operações; criação de estratégias de negócios mais consistentes; medição correta do desempenho; melhor cumprimento dos objetivos traçados. Os benefícios podem promover a geração de vantagem competitiva na empresa tomada por objeto de estudo da pesquisa descrita nesse artigo. Kaplan e Norton (2004) advogam que nada pode melhorado se não for medido.

A partir disto, a implementação formal do $P M S$ se mostra promissora ao processo de gestão e no aprimoramento dos sistemas de controle gerenciais, por permitir a identificação de desvios operacionais ou estratégicos, permitindo a partir disto, o ajuste de processos ou de indivíduos que possam estar em desajuste com os objetivos organizacionais. Ademais, outros estudos têm se preocupado coma necessidade de transparência (compliance) e com a prestação de contas (accountability) dos gestores para com os recursos que lhe foram confiados e o PMS tem condições de ser utilizado como base do processo de avaliação de desempenho. A simplicidade da metodologia de implementação também se mostra como um ponto favorável para a escolha desse modelo para a avaliação de desempenho.

Isto posto, é possível concluir que o estudo atingiu seus objetivos por evidenciar quais são os benefícios que o sistema de avaliação de desempenho $P M S$ tem a oferecer para o SCG de uma empresa de calçados, assim como, por evidenciar também quais barreiras foram encontradas no processo de implantação do modelo. Os pontos positivos reforçam a aplicabilidade do modelo. Já os pontos negativos e as barreiras, se mostram como pontos de atenção para outros estudos que venham ser realizados.

Como sugestão para pesquisas futuras, se mostra oportuno comparar as vantagens e desvantagens entre a implementação do $B S C$ e do $P M S$ de modo a evidenciar qual das duas opções é mais adequada para a realidade econômica brasileira.

\section{Referências}

AGUIAR, A. B.; PACE, E. S.; FREZATTI, F. Análise do Inter-relacionamento das Dimensões da Estrutura de Sistemas de Controle Gerencial: um Estudo Piloto. Departamento de Contabilidade e Atuária da FEA/USP, São Paulo - SP., Brasil. 2009.

ALVES, A. B. Desenho e uso dos sistemas de controle gerencial e sua contribuição para a formação e implementação da estratégia organizacional. (Dissertação de mestrado). Universidade de São Paulo - USP, São Paulo - SP., 2010.

ANTHONY, R. N. Contabilidade gerencial: uma introdução à contabilidade. Ed. Atlas: São Paulo - SP., 1976.

ANTHONY, R. N; GOVINDARAJAN V. Sistemas de Controle Gerencial. 12. ed. Ed. Mcgraw Hill Brasil: Porto Alegre - RS., 2008. 
CANDIDO, A. C. Inovação disruptiva: Reflexões sobre as suas características e implicações no mercado. Research Centre on Enterprise and Work Innovation. Faculdade de Ciências e Tecnologia da Universidade de Lisboa. Portugal. 2011.

COLLIS, J.; HUSSEY, R. N. Pesquisa em Administração. 2. ed. Ed. Bookman, Porto Alegre - RS., 2005.

DeLONE, W. H.; McLEAN, E. R. The DeLone and McLean model of information system success: A ten-year update. Journal of Management Information Systems, v. 19, n. 4, Spring, 2003.

DRUKER, P. F. Managements new role. Coleção Harvard de Administração. Ed. Nova Cultural. São Paulo - SP., 1986.

FLAMHOLTZ, E. Effective organizational control: A framework, applications, and implications. European Management Journal. v.14. n. 6, 1996.

GOMES, J. S.; SALAS, J. M. Controle de gestão: uma abordagem contextual e organizacional. Ed. Atlas: São Paulo - SP., 1999.

HERATH, S. K. A framework for management control research. Journal of Management Development, v. 26, n. 9, 2007. DOI: 10.1108/02621710710819366.

HOFSTEDE, G. The Poverty of Management Control Philosophy. The Academy of Management Review, v. 3, n. 3, jul., 1978.

JOHNSON, H. T.; KAPLAN, R. S. Relevance Lost: The rise and fall of management accounting. Harvard Business School Press. 1987.

KAPLAN, R. S. New systems for measurement and control. Tijdschrift Voor Economie en Management, v. XXXVI, 1991.

KAPLAN, R. S.; NORTON, D. P. Kaplan e Norton na prática. Ed. Elsevier, Rio de Janeiro - RJ., 2004.

KINDALL, A. F; GATZA, J. Positive program for performance appraisal. Coleção Harvard de administração. Ed. Nova Cultural. São Paulo - SP., 1986.

LEÃO, A. L. M. S.; MELLO, S. C. B. Institucionalização como mecanismo de controle em marketing. Revista de Administração Contemporânea, v. 14, n. 2, mar/abr., 2010. http://dx.doi.org/10.1590/S1415-65552010000200005

LORANGE, M. S.; MORTON, S. S. Management control systems: A conceptual framework. July, 1974.

MAGRETTA, J. Entendendo Michael Porter: O guia essencial da competição estratégica. Ed. HSM, São Paulo - SP., 2012.

MALMI, T.; BROWN, D. A. Management control systems as a package: Opportunities, challenges and research directions. Management Accounting Research, v. 19, 2008. https://doi.org/10.1016/j.mar.2008.09.003

MARTINS, G. A.; THEÓPHILO, C. R. Metodologia da investigação científica para ciências sociais aplicadas. Ed. Atlas, São Paulo - SP., 2009.

MENDES, D. A. P.; SILVA, C. E.V; SILVA, J.A.; GOMES, J.S. Controle Gerencial em Empresas Brasileiras Internacionalizadas: O Caso do Bob's. In: XXVI Encontro Nacional da 
Associação dos Programas de Pós-graduação em Administração (ENANPAD). Salvador, BA, 2002. Anais... Salvador - BA., ISSN: 2177-2576.

MERCHANT, K. A.; VAN der STEDE, W. A. Management Control Systems: performance measurement, evaluation and incentives. Financial Times, 2001.

MONTGOMERY, C.; PORTER, M. E. Estratégia: A busca pela vantagem competitiva. Ed. Campus, Rio de Janeiro - RJ., 1998.

MORO, J. R. Implantação de um sistema de informações gerenciais numa empresa familiar da área da saúde, com reduzida estrutura. Dissertação de Mestrado. Universidade Presbiteriana Mackenzie. São Paulo, 2014.

OTLEY, D.; FERREIRA, A. The Design and use of management control systems: An extended framework for analysis. In: $2^{\mathrm{a}}$ Conference on Performance Measurement and Management Control, France, 2005.

OTLEY, D.; FERREIRA, A. The Design and use of management control systems: An extended framework for analysis. Management Accounting Research, October, 2005. DOI: $10.2139 /$ ssrn.682984.

OTLEY, D. Performance management: a framework for management control systems research. Management Accounting Research, v.10, n.4, 2009. DOI:

10.1006/mare.1999.0115.

PRAHALAD, C. K.; HAMEL, G. The core competence of the corporation. Harvard Business Review, 1990.

SIMONS, R. Accounting control system and business strategy: an empirical analysis.

Accounting, Organizations and Society, v. 12, Great Britain, 1987.

https://doi.org/10.1016/0361-3682(87)90024-9

SIMONS, R. The role of management control systems in creating competitive advantage: new perspectives. Accounting Organizations and Society, v. 15, Great Britain, 1990. https://doi.org/10.1016/0361-3682(90)90018-P

SIMONS, R. How new top managers use control systems as levers of strategic renewal.

Strategic Management Journal, v. 15, n.3, 1994. DOI: 10.1002/smj.4250150301

SOUSA NETO, J. A.; MARTINS, H. C. Finanças corporativas: Práticas e estudos de caso. Ed. Elsevier Rio de Janeiro - RJ., 2010.

TAVARES, M. C. Gestão estratégica. 2. ed. Ed. Atlas: São Paulo - SP., 2000.

YIN, R. K. Estudo de caso. Planejamento e métodos. 2. ed. Ed. Bookman: Porto Alegre RS, 2001. 